\title{
Ising antiferromagnet with mobile, pinned, and quenched defects
}

\author{
W.Selke, M.Holtschneider, R.Leidl \\ Institut für Theoretische Physik, \\ Technische Hochschule, \\ 52056 Aachen, Germany
}

Received November 9, 2004

Motivated by recent experiments on $(\mathrm{Sr}, \mathrm{Ca}, \mathrm{La}){ }_{14} \mathrm{Cu}_{24} \mathrm{O}_{41}$, a two-dimensional Ising antiferromagnet with mobile, locally pinned and quenched defects is introduced and analyzed using mainly Monte Carlo techniques. The interplay between the arrangement of the defects and the magnetic ordering as well as the effect of an external field are studied.

Key words: Ising model, randomness, Monte Carlo, cuprates

PACS: 05.10.Ln, 05.50+q, 74.72.Dn, 75.10.Hk

\section{Introduction}

Several interesting low-dimensional magnetic properties arise from the $\mathrm{CuO}_{2}$ chains in $(\mathrm{Sr}, \mathrm{La}, \mathrm{Ca})_{14} \mathrm{Cu}_{24} \mathrm{O}_{41}$. Pertinent experimental findings [1-4] motivated recent theoretical studies on two-dimensional Ising antiferromagnets with defects [5-8].

In particular, a simple Ising model on a square lattice with mobile defects has been introduced [5], with the chain direction corresponding to one of the axes of the lattice. The spins, $S_{(i, j)}= \pm 1$ at lattice site $(i, j)$, correspond to the magnetic $\mathrm{Cu}^{2+}$ ions, and the defects, $S_{(i, j)}=0$, to those $\mathrm{Cu}$ ions which are believed to be spinless due to holes (Zhang-Rice singlets). In the model, the neighbouring spins are supposed to be ferromagnetically coupled in each chain and antiferromagnetically in adjacent chains. In addition, each pair of next-nearest neighbour spins in the same chain separated by a defect is presumed to interact strongly antiferromagnetically. The (mobile) defects are allowed to hop to neighbouring sites in the chains, with the energy barriers of these moves given merely by the magnetic couplings.

Without defects, the model describes a two-dimensional Ising antiferro- or metamagnet, with ferromagnetic ordering in the chains and antiferromagnetic ordering between the chains in the low-temperature phase [9]. The defects form, at low temperatures, nearly straight stripes, perpendicular to the $\mathrm{CuO}_{2}$ chains, separating antiferromagnetic domains. The coherency of the stripes gets lost at a phase transition of first order $[5,6]$. 
To mimic the possible pinning of the defects, due to the La ions, variants of the model may be considered with local pinning positions at periodic $[6,7]$ or random sites. For indefinitely large pinning strength, the defects will be quenched at fixed sites. Obviously, pinning or even quenching may affect the stability of the defect stripes, the magnetic ordering, and related phase transitions.

Note that the model, albeit being motivated experimentally, is thought to be of genuine theoretical interest as well, describing the interplay of magnetic structures and defect arrangements. In that respect, the analysis of the model belongs to the intriguing studies dealing with various aspects on randomness in magnets, ranging, say, from site diluted ferromagnets to spin glasses [10-15].

Concerning the interpretation of experiments on $(\mathrm{Sr}, \mathrm{La}, \mathrm{Ca}){ }_{14} \mathrm{Cu}_{24} \mathrm{O}_{41}$, attention may be also drawn to current theoretical analyses based on two-dimensional anisotropic Heisenberg models [3,16,17].

The article is organized as follows: In the next section, the Ising model with defects is introduced. Results are discussed in section 3, reviewing as well as illustrating previous, recent findings and presenting new results on the "full model" with quenched defects. A brief summary concludes the paper.

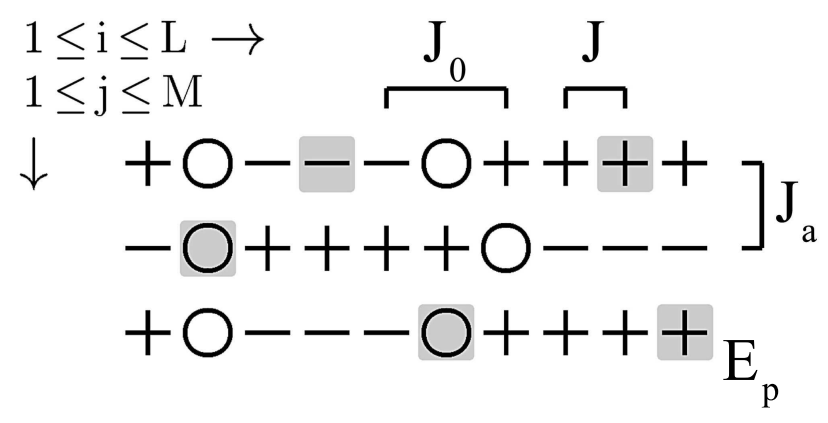

Figure 1. Sketch of the model. Pinning sites are indicated by shadowed squares.

\section{Model}

The Ising model with mobile, pinned or quenched defects is defined on a square lattice with one axis corresponding to the chain direction, say, the horizontal, $i$-axis, as shown in figure 1. Each lattice site, $(i, j)$, is occupied either by a spin, $S_{(i, j)}= \pm 1$, or by a defect, $S_{(i, j)}=0$. The concentration of defects is fixed to be ten percent of the lattice sites, as it seems to be the case in $\mathrm{La}_{5} \mathrm{Ca}_{9} \mathrm{Cu}_{24} \mathrm{O}_{41}$. For simplicity, we shall assume the same concentration of defects in each chain. The interactions, as sketched in figure 1, are as follows. Neighbouring spins are coupled ferromagnetically, $J>0$, along the chains, and antiferromagnetically, $J_{\mathrm{a}}<0$, perpendicular to them. In addition, next-nearest neighbouring spins in the same chain separated by a defect interact antiferromagnetically, $J_{0}<0$, as suggested by experiments. A local pinning potential, $E_{\mathrm{p}}\left(i_{\mathrm{p}}, j_{\mathrm{p}}\right)$ at fixed sites $\left(i_{\mathrm{p}}, j_{\mathrm{p}}\right)$ may act upon defects, reducing the energy 
by the pinning strength. Here we shall assume that the concentration of pinning sites is identical to the concentration of defects, with the same number of pinning sites and defects in each chain. Furthermore, the pinning strength $E_{\mathrm{p}}$ is taken to be the same at each pinning site. If $E_{\mathrm{p}}=0$, the defects are called mobile, at finite values of $E_{\mathrm{p}}>0$, they are pinned, and at $E_{\mathrm{p}} \rightarrow \infty$, the defects are quenched. For finite pinning strength, defects are allowed to diffuse along the chains, keeping a minimal distance of two lattice spacings.

When $J$ and $\left|J_{0}\right|$ are large compared to $\left|J_{\mathrm{a}}\right|$, as suggested by experiments, one may arrive at the 'minimal version' of the model, where spins along a chain are assumed to have the same sign between two defects, reversing sign at a defect. The only relevant energy parameter is then $J_{\mathrm{a}}[5]$.

In the following, we shall present properties of the minimal model with mobile defects and defects pinned at periodic pinning lines perpendicular to the chains, extending recent work $[5-8,18]$.

The "full model", where all couplings are finite, will be discussed without pinning as well as with defects quenched at periodic pinning lines and at random sites. In the following, we take $J_{\mathrm{a}}=-0.3 \mathrm{~J}$ and $J_{0}=-6.25 \mathrm{~J}$, choices motivated by experimental input [5].

Analyses are based on ground state considerations, the free-fermion method to describe thermal properties at low temperatures, the transfer matrix approach, and Monte Carlo simulations.

Simulations are rather demanding, because of, usually, slow fluctuations due to the defects. Typically, the runs over at least $10^{6}$ Monte Carlo steps per site were performed, averaging then over several realizations, simulating lattices with linear dimension $L$ in the $i$ direction, and $M$ in the $j$ direction, see figure 1 . In the following we take $L=M$. In the case of pinning at random sites, an ensemble average has to be taken. Finally, different system sizes have to be simulated especially to accurately determine the phase transition temperatures.

Physical quantities of interest $[5,6]$ include the specific heat, $C$, the susceptibility, $\chi$, and spin correlation functions parallel to the chains,

$$
G_{1}(i, r)=\left(\sum_{j}\left\langle S_{i, j} S_{i+r, j}\right\rangle\right) / M
$$

and perpendicular to the chains,

$$
G_{2}(i, r)=\left(\sum_{j}\left\langle S_{i, j} S_{i, j+r}\right\rangle\right) / M .
$$

Without pinning as well as in the case of defects quenched at random sites, the defect positions are expected to be uncorrelated, so that there is translational invariance with the spin correlations not depending on $i$. Note that in the thermodynamic limit $(L, M \rightarrow \infty)$ for infinitely large distance $r$ the correlations determine the (sublattice) magnetization. 
We also calculated less common microscopic quantities which describe the stability of the defect stripes and the ordering of the defects in the chains $[5,6]$, including the average minimal distance $d_{\mathrm{m}}$ between each defect in chain $j$, at position $\left(i_{\mathrm{d}}, j\right)$, and those in the next chain, at $\left(i_{\mathrm{d}}^{\prime}, j+1\right)$, and the cluster distribution $n_{\mathrm{d}}(l)$ denoting the probability of a cluster with $l$ consecutive spins of equal sign in a chain (as considered, e.g., in percolation theory [19]). Our main emphasis will be on pairs of defects with $l=1$. Finally, it turned out to be quite useful to visualize the microscopic spin and defect configurations as encountered during the simulation.

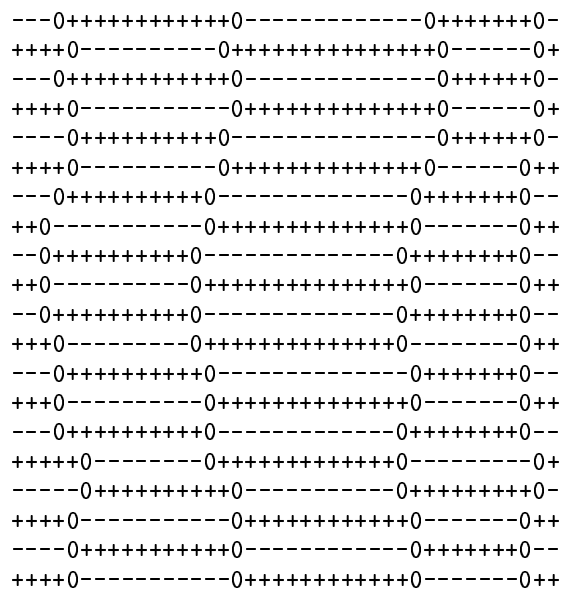

(a)

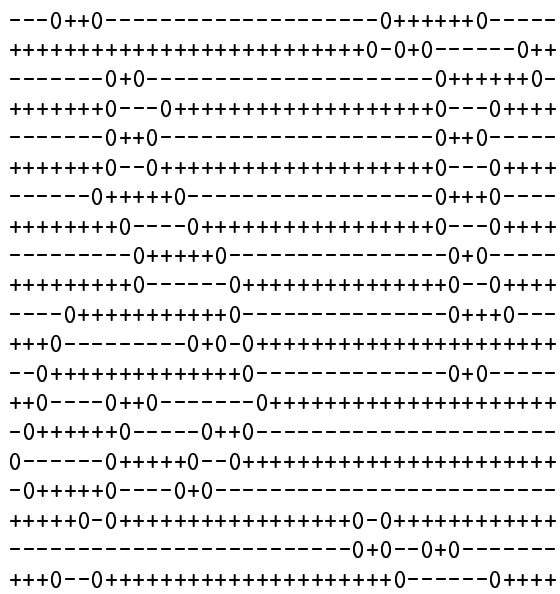

(b)

Figure 2. Typical Monte Carlo equilibrium configurations of the minimal model without pinning at $k_{\mathrm{B}} T /\left|J_{\mathrm{a}}\right|=$ (a) 0.8 and (b) 2.5. Systems with $L=M=40$ were simulated, but only parts are shown. The transition occurs at $k_{\mathrm{B}} T /\left|J_{\mathrm{a}}\right| \approx 1.05[5]$.

\section{Results}

We first present briefly some main results on the minimal model $[5-7,18]$, illustrating crucial features by showing Monte Carlo data for typical equilibrium configurations, see figure 2 , and for correlation functions.

The model with mobile defects, $E_{\mathrm{p}}=0$, is known to form, at $T=0$, straight defect stripes perpendicular to the chains with arbitrary separation between the stripes. Correlations along the chains, $G_{1}$, oscillate, and the amplitude decays exponentially due to the large degeneracy of the ground state (compare figure 3$)$. On the other hand, perpendicular to the chains the spins are fully correlated, $G_{2}(i, r)=0.9(-1)^{r}$, reflecting the antiferromagnetic ordering. As temperature $T$ is increased the stripes will meander, tending to keep, caused by entropic repulsion, on average their largest possible distance. The amplitude of the correlations decays to zero with distance $r$ algebraically, i.e. there is no long-range antiferromagnetic order. At a phase transi- 


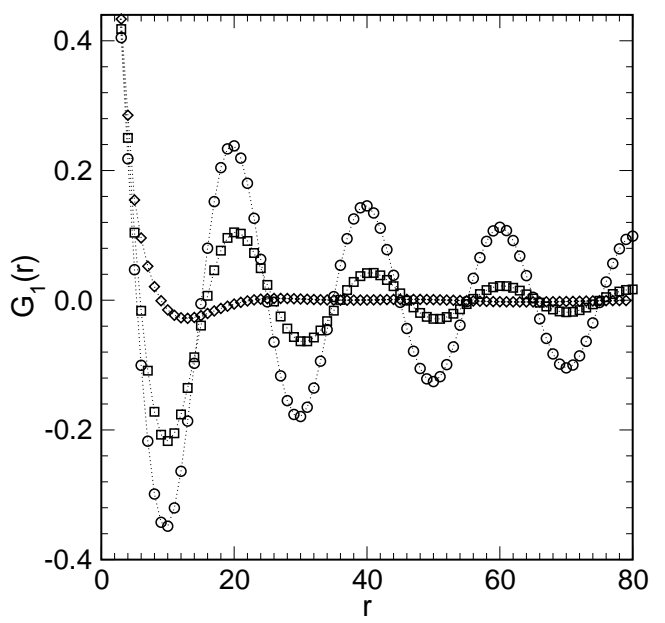

(a)

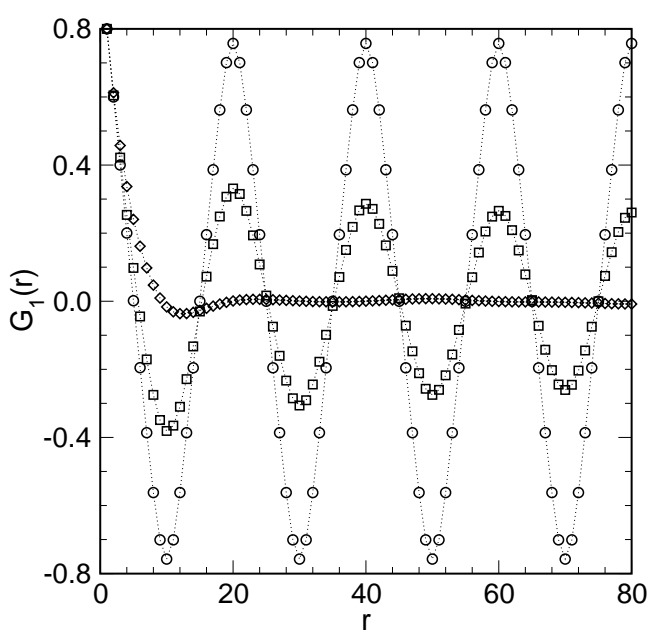

(b)

Figure 3. Correlation function along the chain direction, $G_{1}(r)$, averaging $G_{1}(i, r)$, equation (1), over sites $i$, of the minimal model (a) without pinning, at $k_{\mathrm{B}} T /\left|J_{\mathrm{a}}\right|=0.9$ (circles), 1.3 (squares), and 1.7 (diamonds), and (b) with periodic pinning lines perpendicular to the chains at $k_{\mathrm{B}} T /\left|J_{\mathrm{a}}\right|=1.4$ (circles), 1.8 (squares) and 2.0 (diamonds). In the pinned case, $E_{\mathrm{p}}=\left|J_{\mathrm{a}}\right|$, the transition occurs at $k_{\mathrm{B}} T /\left|J_{\mathrm{a}}\right| \approx 1.5$. Systems with $L=M=160$ were simulated.

tion of first order, the stripes will break up, accompanied by a pairing of defects.

The pairing of the defects, as monitored in the temperature dependence of the probability of next-nearest defect pairs $n_{\mathrm{d}}(1)$, results from an attractive effective interaction between neighbouring defects in a chain. This interaction, mediated by the magnetic coupling $J_{\mathrm{a}}$, occurs for strongly fluctuating stripes [5].

Let us now introduce, in the minimal model, a local pinning, $E_{\mathrm{p}}>0$, of the defects at the sites of straight equidistant lines perpendicular to the chains. Then, at low temperatures, the stripes stay close to these pinning lines and long-range antiferromagnetic order is observed. The phase transition remains to be of first order, driven, again, by the enhanced pairing of defects [6]. Certainly, the transition shifts to higher temperatures as $E_{\mathrm{p}}$ is increased.

The distinction between the algebraic and long-range order at low temperatures without and with pinning is illustrated in figure 3. Here, correlations along the chains, $G_{1}$, are depicted at temperatures below, close to, and above the transition temperature. At low temperatures for $E_{\mathrm{p}}=0$, the height of the maxima in the correlations falls off even at large distance $r$, figure $3 \mathrm{a}$, while it reaches quickly a non-zero constant value in the pinned case $E_{\mathrm{p}}>0$, see figure $3 \mathrm{~b}$.

The impact of an external field on the arrangement of the defect stripes and on the phase transition, both for mobile and periodically pinned defects, has been discussed before $[7,18]$. In particular, at low temperatures, the defect stripes are 
straight at low fields, acquire a zig-zag structure at larger fields and break up into defect pairs when further increasing the field.

The effect of random pinning sites on thermal properties of the minimal model has not been studied in detail yet. Of course, the limiting case of defects quenched at random sites is rather trivial, where the amplitude of the correlations decays exponentially along and perpendicular to the chain, independent of temperature. On the other hand, periodic quenching leads to full antiferromagnetic order at any temperature in the minimal model.

We now turn to the full model with $J_{\mathrm{a}}=-0.3 J$ and $J_{0}=-6.25 J$. The two spins next to a defect still tend to have different sign, because $J_{0}$ is assumed to be rather large. However, spins between two defects may flip quite easily, with the flip energy being determined by the intrachain coupling $J$. Indeed, those quasi one-dimensional spin excitations may mask the phase transition in some thermodynamic quantities, as may be seen, for instance, in the specific heat $[5,18]$. However, by analyzing microscopic quantities describing the stability of the defect stripes, like the minimal distance $d_{\mathrm{m}}$ and the probability of encountering defect pairs separated by merely one spin $n_{\mathrm{d}}(1)$, in the full model with mobile defects, one observes again the phase transition driven by the stripe instability due to defect pairing at a temperature similar to the one in the minimal model $[5,18]$.

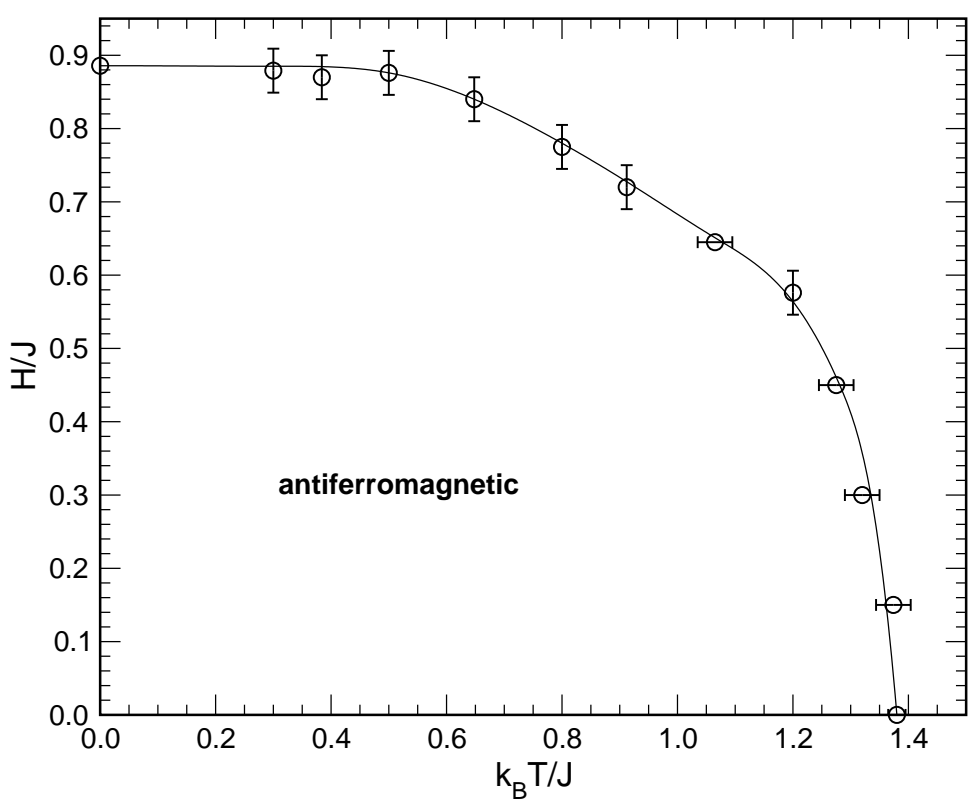

Figure 4. Simulated phase diagram of the full model, taking $J_{\mathrm{a}}=-0.3 J$ and $J_{0}=-6.25 \mathrm{~J}$, with defects quenched at periodic pinning lines perpendicular to the chains, $E_{\mathrm{p}} \rightarrow \infty$. An external field, $H$, is applied.

Introducing quenched defects in the full model, quite interesting thermal properties are observed due to the spin excitations, in contrast to the trivial situation in the minimal model. 
In the case of quenching the defects at periodically placed lines perpendicular to the chains, we determine, using standard Monte Carlo techniques, the phase diagram in the (temperature, field)-plane, as shown in figure 4. In the ground state, when applying and increasing the field, $H$, the antiferromagnetic configuration eventually transforms into a (predominantly) ferromagnetic structure. For sufficiently strong antiferrogmagnetic coupling at the defects, $J_{0}$, as it is the case here, one of the two spins next to a defect will still point against the direction of the field (so that the antiferromagnetic ordering next to a defect is still preserved), while all other spins are aligned parallel to the field. Of course, further increase of the field, at $T=0$, will lead to full ferromagnetic order even for strong couplings $J_{0}$ [18].

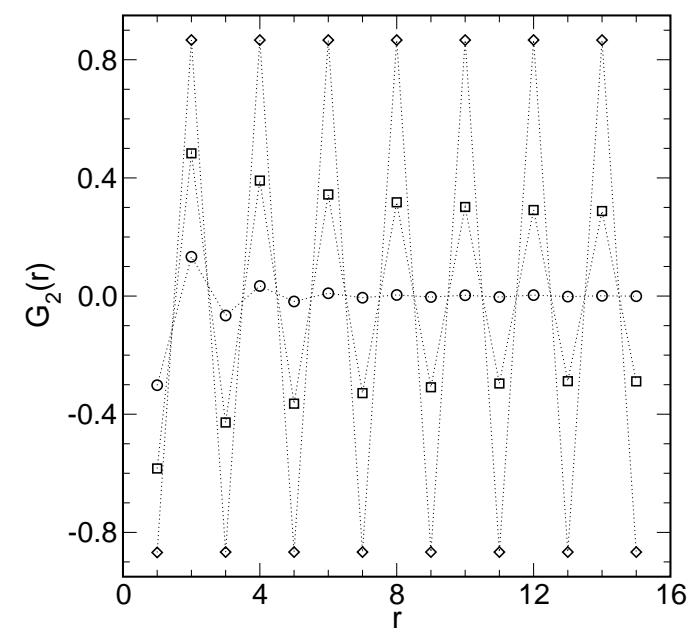

(a)

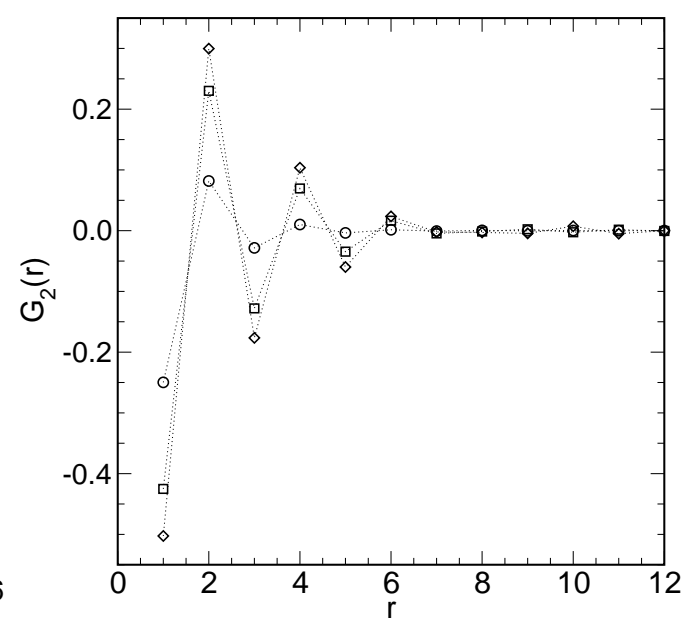

(b)

Figure 5. Correlation function perpendicular to the chain direction $G_{2}(r)$, averaging $G_{2}(i, r)$, equation (2), over sites $i$, of the full model without external field, $H=0$, where quenched defects are (a) at equidistant pinning lines perpendicular to the chains, at temperatures above $\left(k_{\mathrm{B}} T / J=1.8\right.$; circles), close to $(1.4$; squares) and below (1.0; diamonds) the transition temperature (see figure 4 ), and (b) at random sites, above $\left(k_{\mathrm{B}} T / J=1.5\right.$; circles), close to (1.0; squares) and below (0.5; diamonds) the location of the maximum in the specific heat (see figure 6). Lattices with $L=M=40$ sites have been simulated.

While at zero field, $H=0$, the phase transition temperature may be determined exactly by analytical means [20], Monte Carlo simulations are useful to map the entire phase diagram. Here, we obtained the phase transition line by finite-size analyses on data for the specific heat as well as the sublattice magnetization. Note that one encounters, of course, long-range antiferromagnetic ordering in the low-temperature and low-field phase, as observed, e.g., in the correlation function perpendicular to the chains, see figure $5 \mathrm{a}$.

When quenching defects at random sites, the antiferromagnetic order seems to be destroyed even at zero temperature. Ground state analyses for finite systems indicate that the amplitude of the correlations, equations (1) and (2), falls off ex- 
ponentially with large distance $r$. However, the behaviour deviates quantitatively from that of the minimal model with randomly quenched defects. Indeed, at $T=0$, to minimize the energy of the full model, an antiferromagnetic arrangement of the spins in adjacent chains suppressed locally in the minimal model may be partly restored by turning over spins between two consecutive defects. The amplitudes of the simulated spin correlations are observed to decay rapidly with $r$ even at very low temperatures, as illustrated in figure 5b. In fact, there is no evidence for a transition from an algebraically or even long-range ordered antiferromagnetic low-temperature phase to a disordered phase. Instead, the disordered phase seems to extend down to $T=0$.

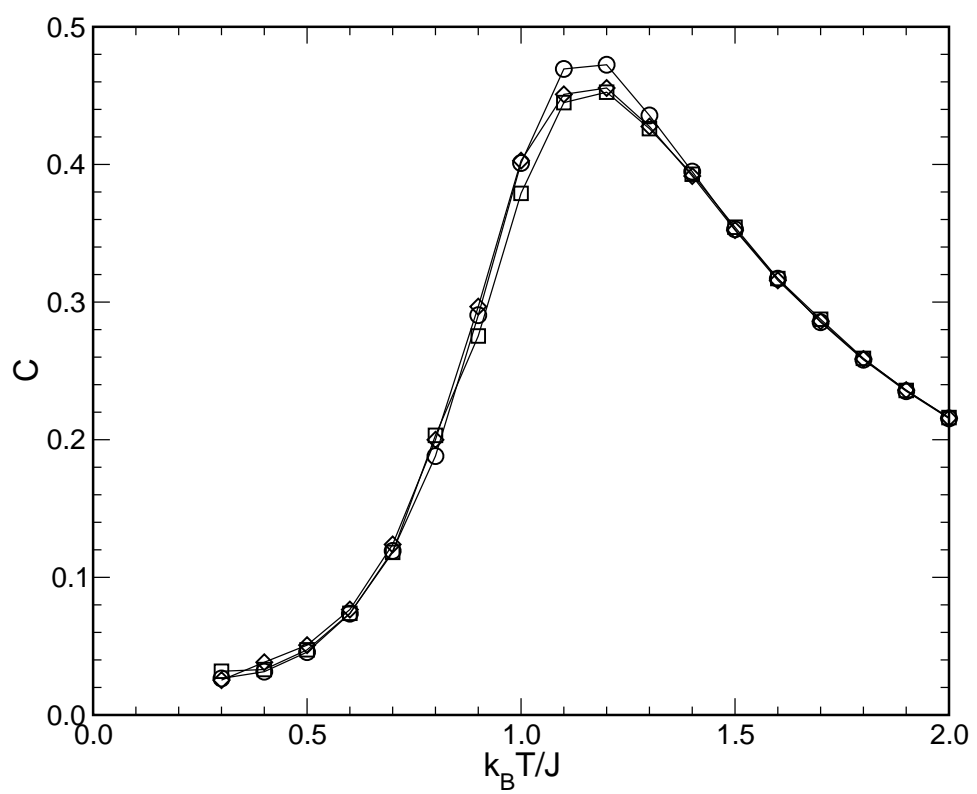

Figure 6. Specific heat $C$ vs. temperature for the full model with quenched defects at random sites. Systems with $L=M=20$ (circles), 40 (squares) and 80 (diamonds) have been simulated, averaging over ensembles of 40, 20, and 10 realizations.

The absence of a phase transition is also reflected in the temperature dependence of the specific heat $C$. As depicted in figure $6, C$ displays a Schottky-type maximum at $k_{\mathrm{B}} T / J \approx 1$, whose height depends very weakly on the system size. Around that temperature, short-range spin correlations get reduced significantly, due to the thermally enhanced number of spin flips.

\section{Summary}

In this article, we studied a two-dimensional Ising model with ferromagnetic interactions between nearest neighbouring spins along one axis, the chain direction, and somewhat weaker antiferromagnetic couplings between spins in adjacent chains. 
The model has an antiferro- or metamagnetic low-temperature phase, with a continuous transition to the disordered phase.

Introducing mobile, pinned or quenched defects with a strong antiferromagnetic interaction between next-nearest neighbour spins in a chain separated by a defect leads to a variety of interesting features, both in the minimal and in the full models.

In both models, mobile defects tend to form stripes at low temperatures. At a phase transition of first order the stripes become unstable, loosing their coherency. The antiferromagnetic ordering in the low-temperature phase is reduced, characterized by algebraically decaying spin correlations.

Long-range order for antiferromagnetic domains may be restored by pinning the defects at (almost) straight lines perpendicular to the chains.

Obviously, properties of the minimal model with quenched defects do not depend on temperature. By quenching the defects in the full model at random sites, the magnetic ordering at low temperatures is destroyed, and we find no evidence for a phase transition.

\section{Acknowledgements}

We thank B.Büchner, R.Klingeler, T.Kroll, and V.L.Pokrovsky for very useful cooperation and information on the topic of this contribution. Financial support by the Deutsche Forschungsgemeinschaft under grant No. SE324 is gratefully acknowledged. One of us (W.S.) thanks Reinhard Folk for numerous enjoyable discussions on statistical physics and antique books.

\section{References}

1. Mizuno Y., Tohyama T., Maekawa S., Osafune T., Motoyama N., Eisaki H., Uchida S., Phys. Rev. B, 1998, 57, No. 9, 5326-5335.

2. Ammerahl U., Büchner B., Kerpen C., Gross R., Revcolevschi A., Phys. Rev. B, 2000, 62, No. 6, R3592-R3595.

3. Matsuda M., Kakurai K., Lorenzo J.E., Regnault L.P., Hiess A., Shirane G., Phys. Rev. B, 2003, 68, No. 6, 060406.

4. Klingeler R. PhD thesis, RWTH Aachen, 2003.

5. Selke W., Pokrovsky V.L., Büchner B., Kroll T., Eur. Phys. J. B, 2002, 30, No. 1, 83-92.

6. Holtschneider M., Selke W., Phys. Rev. E, 2003, 68, No. 2, 026120.

7. Holtschneider M., Leidl R., Selke W., J. Mag. Magn. Mat., 2005, 290-291, 326-329.

8. Kroll T., Klingeler R., Geck J., Büchner B., Selke W., Hücker M., Gukasov A., J. Mag. Magn. Mat., 2005, 290-291, 306-309.

9. Rottman C., Phys. Rev. B, 1990, 41, No. 4, 2547-2549.

10. Binder K., Young A.P., Rev. Mod. Phys., 1986, 58, No. 4, 801-976.

11. Selke W., Shchur L.N., Talapov A.L., Annual Reviews of Computational Physics, 1994, 1, 17-54.

12. Folk R., Holovatch Y., Yavorskii T., Physics-Uspekhi, 2003, 46, No. 2, 169-191. 
13. Berche P.E., Chatelain C., Berche B., Janke W., Eur. Phys. J. B, 2004, 38, No. 3, 463-474.

14. Calabrese P., Parruccini P., Pelissetto A., Vicari E., Phys. Rev. E, 2004, 69, No. 3, 036120 .

15. Skepnek R., Vojta T., Phys. Rev. B, 2004, 69, No. 17, 174410.

16. Leidl R., Selke W., Phys. Rev. B, 2004, 69, No. 5, 056401.

17. Leidl R., Selke W., Phys. Rev. B, 2004, 70, No. 17, 174425.

18. Holtschneider M. Diploma thesis, RWTH Aachen, 2004.

19. Stauffer D., Aharony A. Introduction to Percolation Theory. Taylor and Francis, London, 1994.

20. Igloi F., Peschel I., Turban L., Adv. Phys., 1993, 42, No. 6, 683-740.

\title{
Ізингівський антиферомагнетик із рухомими, закріпленими та замороженими дефектами
}

\author{
В.Зельке, М.Гольцшнайдер, Р.Ляйдл \\ Інститут теоретичної фізики, \\ Вища технічна школа, \\ 52056 Аахен, Німеччина
}

Отримано 9 листопада 2004 p.

У зв'язку 3 недавніми експериментами на $(\mathrm{Sr}, \mathrm{Ca}, \mathrm{La}){ }_{14} \mathrm{Cu}_{24} \mathrm{O}_{41}$, представлено модель двовимірного Ізингівського антиферомагнетика із рухомими, локально закріпленими та замороженими дефектами та проаналізовано її, в основному технікою Монте Карло. Досліджено взаємозв'язок між впорядкуванням дефектів та магнітним впорядкуванням, а також ефект зовнішнього поля.

Ключові слова: модель Ізинга, безлад, Монте Карло, купрати

PACS: $05.10 . \mathrm{Ln}, 05.50+q, 74.72 . \mathrm{Dn}, 75.10 . \mathrm{Hk}$ 